\title{
Mejora en el proceso de pruebas de metales en una empresa de servicio de inspección y laboratorio empleando simulación con ProModel
}

\section{Germán Herrera Vidal}

Docente investigador programa de Ingeniería Industrial, Facultad de Ingeniería, Grupo de investigación CIPTEC, Fundación Universitaria Tecnológico Comfenalco, Cartagena de Indias, Colombia. herreravg@tecnocomfenalco.edu.co

Recibido: 15 Febrero 2013 - Revisado: 30 Abril 2013

Aceptado: 30 Mayo 2013 - Publicado: 30 Junio 2013

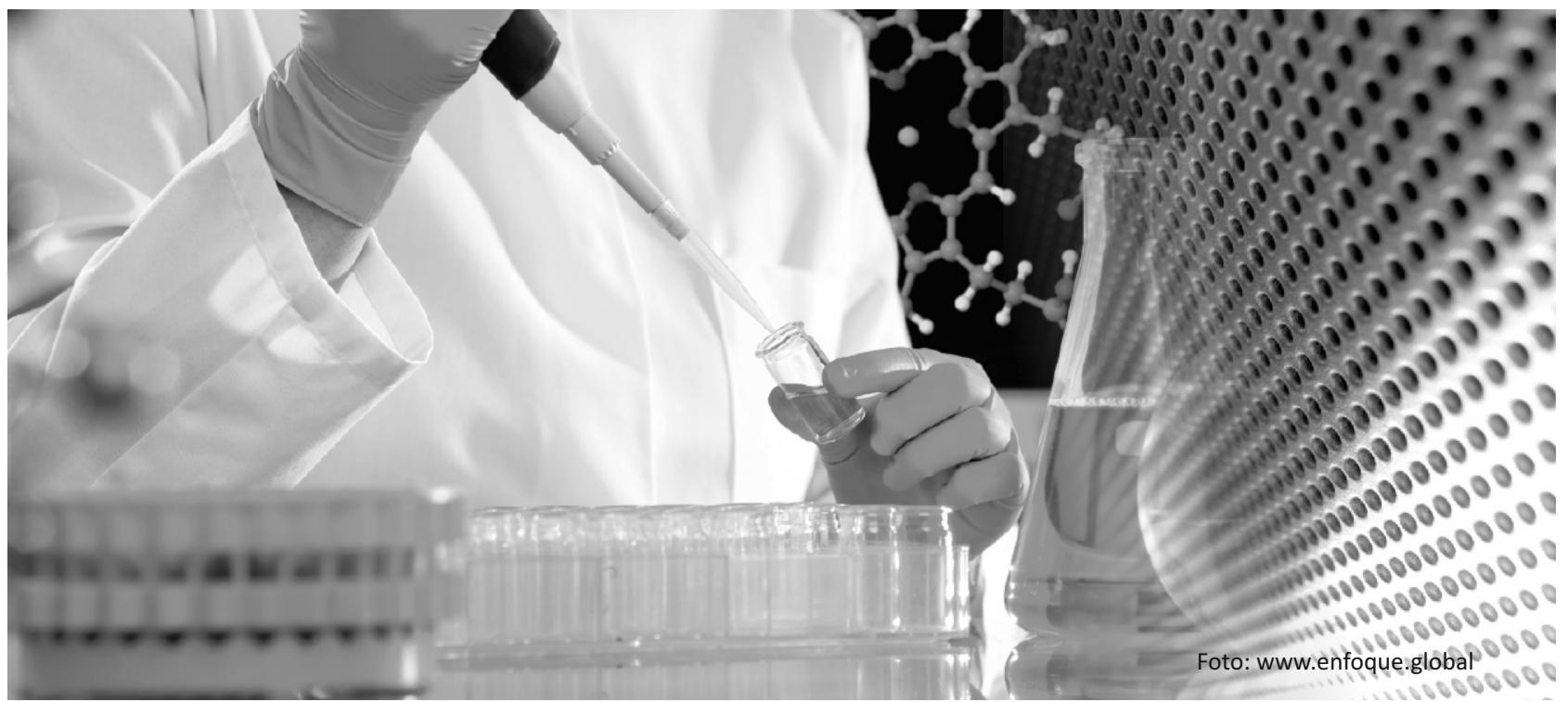

Resumen: En las empresas hoy en día, se hace necesario el uso de tecnologías y herramientas que permitan estudiar los procesos, de acuerdo al comportamiento del mercado de forma eficiente, rápida y económica. En el presente documento, se desarrolla la aplicación de la simulación con ProModel, con el fin de mejorar el proceso de pruebas de metales sílice y aluminio, en una empresa de servicio de inspección y de laboratorio de productos petroquímicos y petróleos. Para esto, se hace necesario metodológicamente describir el proceso productivo, determinar el modelo conceptual y la recolección de datos de entrada, construir el modelo, analizar los resultados de salida y, por último, plantear una mejora, a través del análisis del sistema y de indicadores del proceso, que permita incrementar significativamente el nivel de servicio.

Palabras clave: Sistema, Modelo, Simulación, Mejora de procesos.

Abstract: In companies today, using technologies and tools to study processes, according to market performance efficiently, quickly and cost becomes necessary. In this paper the application of simulation ProModel develops, in order to improve the testing process silica and aluminum metal in a service company and laboratory inspection of petrochemicals and petroleum. For this it is necessary to methodologically clearly describe the production process, determine the conceptual model and gathering input data, build the model, analyze the output results and finally plating improvement, through system analysis and indicators process, which allows to significantly increase the level of service.

Keywords: System, Model, Simulation, Process Improvement. 


\section{INTRODUCCIÓN}

Hoy en día, las empresas están en la obligación de tener sus procesos productivos de manera eficiente y eficaz si quieren mantenerse vigentes en un mercado cada vez más competitivo y globalizado, por ende, la aplicación de herramientas que permitan obtener mejoras en sus procesos, solución a los problemas y satisfacción a los clientes, no se pueden obviar. La simulación precisamente, se utiliza para realizar representaciones de sistemas productivos y a la vez, poder estudiar cómo es el flujo de proceso; de esta manera, se permite realizar permutaciones con el objetivo de analizar el comportamiento y obtener una visión global de los cambios locales realizados en el sistema.

La simulación surge a partir de la evolución del Método de Montecarlo, gracias a la construcción de los primeros computadores de propósito general como el ENIAC; de igual forma, debido a la aplicación de modelos estadísticos y matemáticos por medio de herramientas informáticas. El desarrollo de este trabajo propone el uso del paquete de simulación ProModel, que posibilita construir y analizar el comportamiento del proceso y una de sus herramientas denominada Stat::Fit, con el fin de poder analizar los datos de entradas.

Ante el auge alcanzado por las herramientas, modelos y casos de aplicación en diversas áreas, surge la iniciativa de diseñar unas propuestas de mejora basados en el uso de la simulación. Para esto, en una segunda parte, se especifican los fundamentos teóricos e investigativos del proyecto; en una tercera instancia, los elementos metodológicos desarrollados; en una cuarta, el caso práctico de aplicación y, por último, las conclusiones.

\section{FUNDAMENTOS TEÓRICOS E INVESTIGATIVOS}

\subsection{Sistema}

El concepto de sistemas ha sufrido múltiples utilizaciones y dependiendo de ello, se ha obtenido diferentes definiciones. Prawda, J. (1980), define al sistema como: "Un agregado o conjunto de objetos que interactúan entre sí como una unidad, para la consecución de un propósito explícito o implícitamente definido" (Autor, Año, p.).

De igual forma Gordon, G. (1978), el sistema también se precisa como: "Un agregado o conjunto de objetos reunidos en alguna interacción o interdependencia regular" (Autor, Año, p.).

Según Gigch, J. (1974), el planteamiento sistemático nació para satisfacer las siguientes necesidades: (i) Generalizar, (ii) Simplificar, (iii) Integrar, (iv) Optimizar, (v) Evaluar, (vi) Planificar y (vii) Controlar. Más recientemente, Wiston, W. (2008), define el sistema como: "Una colección de entidades que actúan e interactúan hacia la realización de algún fin lógica" (Autor, Año, p.).

Esta concepción se ajusta a cualquier tipo de sistema, es decir que su aplicación es en todas las ciencias y disciplinas: Ciencias Sociales, Ciencias Humanas, Sistemas Biológicos, entre otros.

Según Gordon, G. (1978), al estudiar los diferentes tipos de sistemas, se van a apreciar determinados objetos distintos, dentro de los cuales, cada uno tiene propiedades de interés. De acuerdo esta última concepción, se manejan términos como "entidad", para denotar un objeto de interés en un sistema, y "atributo", para denotar la propiedad de una entidad. Además, plantea que todo proceso que provoque cambios en el sistema se conocerá como "actividad" y se maneja el término "estado" para indicar una descripción de todas las entidades, atributos y actividades de acuerdo con su existencia en algún punto del tiempo. Por ejemplo, el estado de una oficina bancaria en un instante se podría definir mediante el número de cajeros en él, el número de clientes, el instante de llegada de cada cliente y el tipo de operación que desea realizar cada uno. 
Para realizar un estudio sobre un sistema, es necesario el conocimiento de algunos conceptos como entidad, entidades permanentes, atributos, relación, recursos, estado y evento, los cuales se detallan a continuación:

Entidad: Según García, E., et al. (2006), es la representación de los flujos de entrada a un sistema; este es el elemento responsable de que el estado del sistema cambie. Ejemplo de entidades pueden ser los clientes que llegan a la caja de un banco, las piezas que llegan a un proceso o el embarque de piezas que llegan a un inventario.

Entidades temporales: De acuerdo a lo expuesto por Guash, A. et al. (2005), son todos aquellos objetos que se procesan en el sistema, como por ejemplo: las piezas en un sistema de manufactura, los clientes en un banco.

Actividades: Son las tareas o acciones que tienen lugar en el sistema. Están encapsuladas entre dos eventos. Verbigracia, la reparación de una máquina, el procesado de una pieza o el transporte de un cliente. Según Guash, A. et al. (2005), las actividades generalmente tienen duración temporal y, normalmente, precisan del uso de recursos.

Atributos: Según Barceló, J. (1996), son un conjunto de propiedades que caracterizan a las entidades que forman parte del sistema, por ejemplo: cantidades de pedidos, número de máquinas de un tipo dado, color, prioridad, etc.

Relación: Según Hoeger, H. (2000), es la manera en que las propiedades de una o más entidades dependen entre sí; de esta manera, hacen que un cambio en la propiedad de una entidad ocasione un cambio en una propiedad de otra entidad; ejemplo: En la entidad: Departamento de Producción, sus cambios pueden afectar a los departamentos de compra y ensamblaje.
Recursos o entidades permanentes: Son una clase de entidades, son los medios para ejecutar las actividades. Según Guash, A. et al. (2005), los recursos definen quién o quiénes ejecutan la actividad. Los recursos presentan características como capacidad, velocidad, averías y reparaciones. Ejemplo de recursos son las máquinas en un sistema de manufactura, los mecanismos de transporte o los operadores.

Estado: De acuerdo con Barceló, J. (1996), son los valores y/o condiciones de los atributos de las entidades en un instante dado, después de suscitarse un evento, de forma que se puede saber si una acción se puede ejecutar o si se puede elegir entre varias. Ejemplo: el número de clientes en una cola, los procesos de manufactura en cada departamento de una fábrica, etc.

•Evento: Según Racero, J. (2004), son los diferentes hechos que ocurren en instantes de tiempo para dar lugar a cambios en el estado del sistema y así, identificar qué es lo que origina el cambio y cuándo se origina. Ejemplo: Una llegada, una salida de una entidad.

\subsection{Modelado del Sistema}

La descripción de las características de interés de un sistema se conoce como modelo del sistema y el proceso de abstracción para obtener esta descripción se conoce como modelado. Según Shannon, R. (1988), modelado es el proceso de construcción de un modelo. Un modelo es una representación de un objeto, sistema, o idea; usualmente, su propósito es ayudar explicar, entender o mejorar un sistema.

Gordon, G. (1978), define un modelo así: "Es el cuerpo de información relativa a un sistema recabado para fines de estudiarlo".

Más recientemente Guasch, A., et al. (2002), plantean varias definiciones: 
"Un modelo se desarrolla siempre a partir de una serie de aproximaciones e hipótesis y, consecuentemente, representa tan sólo parcialmente la realidad. "Un modelo se construye para una finalidad específica y debe ser formulado para que sea útil a dicho fin.

"Un modelo tiene que ser por necesidad un compromiso entre la simplicidad y la necesidad de recoger todos los aspectos esenciales del sistema en estudio".

Para Gordon, G. (1978). , la tarea de obtener un modelo de un sistema se deriva en forma genérica en dos subtareas: la determinación de la estructura del modelo y proporcionar los datos. La determinación de la estructura fija la frontera del sistema e identifica las identidades, atributos y actividades del sistema. Los datos suministran los valores de los atributos que pueden tener y definen la relación involucrada en las actividades.

Según Hoeger, H. (2000), de acuerdo a la realidad que representen y la perspectiva que le dé el analista, los modelos se clasifican en:

(A) Modelos de Tiempo Continuo: Son aquellos en los cuales las variables de estado del sistema evolucionan en el tiempo de forma continua. Se pueden representar mediante ecuaciones diferenciales.

(B) Modelos de Tiempo Discreto: Son aquellos en los cuales el estado del sistema se define sólo para instantes particulares de tiempo, por ejemplo, el número de clientes que visitan el banco un día determinado.

(C) Modelos de Estado Continuo o de Eventos Continuos: Representan sistemas cuyos cambios de estado son graduales. Las variables que intervienen son continuas. Son modelos representados por ecuaciones diferenciales donde una de las variables de estado representa una cantidad que cambia continuamente, por ejemplo, la corriente en una bobina.
(D) Modelos de Estado Discreto o de Eventos Discretos: Son modelos dinámicos, estocásticos y discretos en los que las variables de estado cambian de valor en instantes no periódicos de tiempo, sin estar dirigidos por un reloj. Estos instantes de tiempo se corresponden con la ocurrencia de un evento.

(E) Modelos Determinísticos: Son aquellos donde la solución para determinadas condiciones es única y siempre la misma. Ejemplo de este tipo, son los modelos de optimización lineal, donde la solución está determinada unívocamente por el conjunto de ecuaciones $y / 0$ inecuaciones que se suministran como restricciones.

(F) Modelos Probabilísticos o Aleatorios: Son aquellos donde las características del sistema se representan por variables aleatorias. Por ejemplo, los simuladores de colas, donde las llegadas a estas se produce a través de una función de distribución de probabilidades.

(G) Modelos Estáticos: Utilizados para representar sistemas cuyo estado es invariable a través del tiempo. Por ejemplo: la cantidad de materia en energía.

(H) Modelos Dinámicos: Contrario a los anteriores, en estos las variables de salida sí varían en el tiempo, muy empleados en el área de control, para modelar la dinámica de un sistema físico a través de ecuaciones diferenciales dependientes del tiempo.

(I) Modelos Lineales: Son aquellos cuya salida del sistema se representa por una línea recta, ejemplo de estos son los modelos de regresión lineal.

(J) Modelos No Lineales: Empleados para representar sistemas en donde las salidas no sean lineales, por ejemplo, la solución en el tiempo de una ecuación diferencial de segundo orden.

(K) Modelos Abiertos: Se les llama así si los datos de entrada son externos al modelo e independientes de 
él, ejemplo: los sistemas de cola de un banco donde las llegadas son externas al modelo.

(L) Modelos Cerrados: Estos no disponen de una entrada del exterior; ejemplo de ellos son los modelos de control en su estado natural (No hay entrada presente).

(M) Modelos Estables: Son aquellos donde la variable de salida converge a un valor (independiente del tiempo). Ejemplo: cuando el intervalo de llegada de clientes es mayor que el tiempo de servicio.

(N) Modelos Inestables: Cuando el comportamiento del sistema cambia constantemente; al contrario del anterior, no hay tendencia a un valor estable. Ejemplo de estos modelos son los sistemas de colas en un banco, donde el intervalo entre llegadas es menor que el tiempo de servicio.

\subsection{Simulación}

Tiene sus primicias en 1948 con el trabajo de Harris y Herman Kahn, en los cuales se inicia el estudio de la simulación como campo de conocimiento; estos sistematizaron las primeras técnicas de simulación que hoy en día se han venido aplicando en diferentes entornos. (Aspray, W. y Neumann, J., 1990).

Uno de los pioneros en brindar una definición aceptada y más difundida de la palabra de simulación fue dada por Naylor, B. y Kong, Ch. (1971), quien la define así:

La simulación es una técnica numérica para conducir experimentos en una computadora digital. Estos experimentos comprenden ciertos tipos de relaciones matemáticas y lógicas, las cuales son necesarias para describir el comportamiento y la estructura de sistemas complejos del mundo real a través de largos periodos de tiempo (p. 42)

Basado en la concepción anterior, la cual está en un contexto muy amplio, ya que puede incluir desde el diseño de un prototipo o una maqueta hasta un programa o software sistematizado, Maisel, H. y
Grugnoli, G. (1972), definen la simulación en un sentido más estricto, como:

Una técnica numérica para realizar experimentos en una computadora digital. Estos experimentos involucran ciertos tipos de modelos matemáticos y lógicos que describen el comportamiento de sistemas de negocios, económicos, sociales, biológicos, físicos o químicos a través de largos periodos de tiempo.

Consecuentemente a estas definiciones, Shannon, R. (1975), conciben la simulación como:

Simulación es el proceso de diseñar y desarrollar un modelo computarizado de un sistema o proceso real y conducir experimentos este modelo, con el propósito de entender el comportamiento del sistema o evaluar varias estrategias con las cuales se puede operar el sistema (p. 16).

Hernández, D. (1977), señala que la simulación significa: "Representación de la realidad y por tanto la descripción verbal y la representación esquemática o gráfica de alguna parte del mundo real constituye una simulación" (p. 27).

Otras definiciones de simulación se recogen en Thierauf, R. y Grosse, R. (1976), que definen la simulación como:

"El uso de un modelo de sistema que tiene la característica deseada de la realidad, a fin de reproducir la esencia de las operaciones" (p. 33).

"Una representación de la realidad mediante el empleo de un modelo u otro mecanismo que reaccionará del mismo modo que la realidad bajo una serie de condiciones dadas" (p. 33).

"Una técnica numérica, para realizar experimentos con ciertos tipos de modelos matemáticos, que describen la conducta de sistemas complejos sobre un cierto periodo de tiempo" (p. 34). 
Posteriormente, Banks, J., et al. (1996), consideran la simulación como:

El desarrollo de un modelo lógico matemático de un sistema, de tal forma que se tiene una imitación de la operación de un proceso de la vida real o de un sistema a través del tiempo. La simulación involucra la generación de una historia artificial de un sistema, la observación de esta historia mediante la manipulación experimental, nos ayuda a inferir las características operacionales de tal sistema (p. 8).

Teniendo en cuenta los diferentes modelos de sistemas, las definiciones anteriormente descritas no denotan si los sistemas a modelar son discretos o continuos, especifican si los sistemas modelados son continuos o discretos; es por esto que según García, E., et al. (2006), la simulación de eventos discretos es el conjunto de relaciones lógicas, matemáticas y probabilísticas que integran el comportamiento de un sistema bajo estudio, cuando se presenta un evento determinado. Y de acuerdo con Gordon, G. (1978), un sistema continuo es aquel en que las actividades predominantes del sistema cambian continuamente con el tiempo. Estos modelos suelen usar ecuaciones diferenciales para describir las interacciones entre los distintos elementos del sistema.

La simulación es una herramienta indispensable para el análisis de sistemas, la toma de decisiones y la mejora de procesos. Aparte de esto, Naylor, B. y Kong, Ch. (1971), ha sugerido que un estudio de simulación es muy recomendable, porque presenta las siguientes ventajas:

A través de un estudio de simulación, se puede analizar el efecto de cambios internos y externos del sistema, al hacer alteraciones en el modelo del sistema y observando los efectos de esas alteraciones en el comportamiento del mismo.

Una observación detallada del sistema que se está simulando puede conducir a: un mejor entendimiento de este $y$, por consiguiente, a sugerir estrategias que mejoren la operación y eficiencia del sistema.
La técnica de simulación puede ser utilizada como un instrumento pedagógico para enseñar a estudiantes, habilidades básicas en análisis estadístico, análisis teórico, etc.

La simulación de sistemas complejos puede ayudar a entender mejor la operación del sistema, a detectar las variables más importantes que interactúan en él y a entender mejor las interrelaciones entre las variables.

La técnica de simulación se puede utilizar también para entrenamiento de personal. En algunas ocasiones, se puede tener una buena representación de un sistema (como, por ejemplo, los juegos de negocios) y, entonces, a través de él, es posible entrenar y dar experiencia a cierto tipo de personal.

La técnica de simulación puede ser usada para experimentar con nuevas situaciones, sobre las cuales se tiene poca o ninguna información. A través de la experimentación, nos podemos anticipar mejor a posibles resultados no previstos.

Cuando nuevos elementos son introducidos en un sistema, la simulación puede ser usada para anticipar cuellos de botella o algún otro problema que pueda surgir en el comportamiento de este.

A diferencia de las ventajas mencionadas, según Coss, R. (2003), la técnica de simulación presenta el problema de requerir equipo computacional y recursos humanos costosos. Además, generalmente se requiere tiempo para que un modelo de simulación sea desarrollado y perfeccionado. Finalmente, es posible que la alta administración no entienda esta técnica, lo que crea dificultad paran vender la idea.

Existe una gran cantidad de áreas donde la técnica de simulación puede ser aplicada, según Montoya, J. (2007), la simulación, como una herramienta de soporte dentro del proceso de toma de decisiones, puede ser usada para la planeación y mejoramiento de diferentes áreas dentro del ámbito empresarial de 
manufactura y servicios, como por ejemplo:

Sistemas de líneas de espera: La simulación de eventos discretos permite estudiar y analizar los sistemas de filas o colas de espera cuya representación formal resultara demasiado compleja de analizar a través de una formulación matemática.

Sistema de inventarios: La simulación permite estudiar y comparar políticas para la administración de inventarios en las cuales todos los parámetros (tiempos de entrega, demanda, costo) son de carácter estocástico.

Sistemas de manufactura: Ésta es un área en la cual la simulación ha tenido tradicionalmente gran aceptación, puesto que prácticamente todos los sectores industriales encuentran cabida para la aplicación de modelos de simulación como herramienta de apoyo en el proceso de toma de decisiones. Entre los sectores industriales de mayor aplicación, se encuentran la industria automotriz, la fabricación de circuitos integrados, exploración petrolera, la industria química, la fabricación papelera, la cadena logística global.

Industria de servicios: El área del sector servicios ha recibido mucho apoyo de la simulación para la toma de decisiones. Esta es un poco más compleja de modelar que la fabricación de bienes, debido al carácter intangible del producto servicio. Aplicaciones concretas se encuentran principalmente en el análisis y diseño de centros de llamadas, bancos, supermercados, entre otros.

Proyectos de inversión: La simulación es recomendada para el estudio de proyectos de inversión en los cuales la incertidumbre asociada a la tasa de inflación, las tasas de interés, los flujos de efectivo, etc.; tal incertidumbre hace difícil y a veces imposible analizar analíticamente los flujos de caja.

Sistemas de transporte y distribución: La simulación permite representar y analizar el comportamiento de sistemas de transporte tanto en redes metropolitanas (tráfico en las autopistas o en las ciudades, pertinencia de un semáforo en el cruce de dos vías) como en sistemas de fabricación (manejo y almacenamiento de producto en proceso). Se pueden así comparar varias estrategias para la gestión de los recursos (vehículos) o ayudar al diseño del sistema a través del cálculo del número necesarios o a la configuración de la res de transporte como tal. Igualmente, La distribución de productos a lo largo de la red logística es un área de particular interés debido a la complejidad asociada al proceso.

Según Racero, J. (2004), los campos y áreas de aplicación de la simulación son extensos, sin embargo, se pueden presentar de manera resumida mediante la Tabla 1.

Tabla 1. Campos de aplicación de la simulación.

\begin{tabular}{|c|c|}
\hline Campos & Aplicación \\
\hline Computación & $\begin{array}{l}\text { Redes de ordenadores, componentes, } \\
\text { programación, bases de datos, fiabilidad. }\end{array}$ \\
\hline Manufactura & $\begin{array}{l}\text { Manejo de materiales, líneas de montaje, } \\
\text { equipos de almacenamiento, control de } \\
\text { inventario, mantenimiento, distribución en } \\
\text { planta, diseño de máquinas. }\end{array}$ \\
\hline Negocios & $\begin{array}{l}\text { Análisis de existencias, política de precios, } \\
\text { estrategias de marketing, estudios de } \\
\text { adquisición, análisis de flujo de caja, } \\
\text { predicción, alternativas del transporte, } \\
\text { planificación de mano de obra. }\end{array}$ \\
\hline Gobierno & $\begin{array}{l}\text { Armamento y su uso, tácticas militares, } \\
\text { predicción de la población, uso del suelo, } \\
\text { prevención de incendios, servicios de } \\
\text { policía, justicia criminal, diseño de vías de } \\
\text { comunicación, servicios sanitarios. }\end{array}$ \\
\hline $\begin{array}{l}\text { Ecología y } \\
\text { Ambiente }\end{array}$ & $\begin{array}{c}\text { Contaminación y purificación del agua, } \\
\text { control de residuos, contaminación del } \\
\text { aire, control de plagas, predicción del } \\
\text { tiempo, análisis de sismos y tormentas, } \\
\text { exploración y explotación de minerales, } \\
\text { sistemas de energía solar, explotación de } \\
\text { cultivos. }\end{array}$ \\
\hline $\begin{array}{l}\text { Sociedad y } \\
\text { Comportamiento }\end{array}$ & $\begin{array}{l}\text { Estudios de alimentación de la población, } \\
\text { políticas educativas, estructuras } \\
\text { organizativas, análisis de sistemas sociales, } \\
\text { sistemas de asistencia social, } \\
\text { administración universitaria. }\end{array}$ \\
\hline Biociencias & $\begin{array}{l}\text { Rendimiento en el deporte, control de } \\
\text { epidemias, ciclos de vida biológicos, } \\
\text { estudios biomédicos. }\end{array}$ \\
\hline
\end{tabular}


Haciendo una revisión de la literatura en cuanto a casos de aplicación de la simulación, teniendo en cuenta las diferentes áreas y herramienta informática (Ver Tabla 2), se puede resaltar que una de las herramientas más utilizadas para llevar a cabo modelos de simulación en áreas como manufacturas, servicios, Teoría de Colas o Líneas de Espera es la herramienta ProModel y Arena. Esto no quiere decir que no se hayan utilizados otras, como es el caso del área de proyecto de inversión, donde sobresalen herramientas como Ithink, Siminv o Witness.

Tabla 2. Relación de áreas de aplicación investigadas vs. Herramientas de simulación.

\begin{tabular}{|c|c|c|}
\hline Autores & $\begin{array}{l}\text { Herramienta } \\
\text { Simulación }\end{array}$ & $\begin{array}{c}\text { Área de } \\
\text { Aplicación }\end{array}$ \\
\hline $\begin{array}{l}\text { Álvarez Pomar, L., } \\
\text { y Mejía, G. (2005). }\end{array}$ & ProModel & \multirow{6}{*}{$\begin{array}{l}\text { Teoría de } \\
\text { Colas o } \\
\text { Líneas de } \\
\text { Espera }\end{array}$} \\
\hline Riaño, G. (2007). & Arena & \\
\hline $\begin{array}{c}\text { Portilla, L. et al. } \\
\text { (2010). }\end{array}$ & ProModel & \\
\hline $\begin{array}{c}\text { Paternina, C. et al. } \\
(2011)\end{array}$ & Arena & \\
\hline $\begin{array}{l}\text { Nieto Buitrago, D. y } \\
\text { Osorio Zambrano, } \\
\text { K. (2012). }\end{array}$ & ProModel & \\
\hline Borgetti, S. (2013). & ProModel & \\
\hline $\begin{array}{c}\text { Moraga Rebolledo, } \\
\text { M., y Baesler } \\
\text { Abufarde, F. (2001). }\end{array}$ & Arena & \multirow{9}{*}{$\begin{array}{l}\text { Sistemas de } \\
\text { Manufactur } \\
\text { as }\end{array}$} \\
\hline $\begin{array}{c}\text { De la Fuente García, } \\
\text { D. et al. (2002). }\end{array}$ & Arena & \\
\hline $\begin{array}{c}\text { Flores, F., y } \\
\text { Bernardi, B. (2007). }\end{array}$ & ProModel & \\
\hline $\begin{array}{c}\text { Moran Pacheco, R. } \\
\text { y Torres } \\
\text { Fuchslocher, C. } \\
\text { (2008). }\end{array}$ & ProModel & \\
\hline $\begin{array}{c}\text { Lugo Ruíz, A. y } \\
\text { Pinzón Blanco, D. } \\
\text { (2011). }\end{array}$ & ProModel & \\
\hline Soto, L. (2011). & Petrinet & \\
\hline $\begin{array}{c}\text { Arancibia Vallejos, } \\
\text { C. (2012). }\end{array}$ & ProModel & \\
\hline $\begin{array}{l}\text { Saucedo Solorio, J. } \\
\text { (2013). }\end{array}$ & ProModel & \\
\hline $\begin{array}{l}\text { Cruz, M., Sánchez, } \\
\text { (2013). }\end{array}$ & ProModel & \\
\hline
\end{tabular}

\begin{tabular}{|c|c|c|}
\hline Autores & $\begin{array}{l}\text { Herramienta } \\
\text { Simulación }\end{array}$ & $\begin{array}{c}\text { Área de } \\
\text { Aplicación }\end{array}$ \\
\hline $\begin{array}{c}\text { Rojas, L. y Herrera, } \\
\text { L. (2008). }\end{array}$ & Arena & \multirow{6}{*}{$\begin{array}{l}\text { Sistemas de } \\
\text { Servicios }\end{array}$} \\
\hline $\begin{array}{c}\text { Avila, H. y } \\
\text { Vásquez, M. (2008). }\end{array}$ & Arena & \\
\hline $\begin{array}{c}\text { Díaz, J., Cubillos, } \\
\text { J., y Fernández, M. } \\
\text { (2010). }\end{array}$ & ProModel & \\
\hline $\begin{array}{c}\text { Restrepo, E. et al. } \\
\text { (2011). }\end{array}$ & ProModel & \\
\hline Acosta, E. (2012). & ProModel & \\
\hline $\begin{array}{c}\text { Taddei Bringas, J. et } \\
\text { al. (2013). }\end{array}$ & ProModel & \\
\hline $\begin{array}{c}\text { Álvarez, S. et al. } \\
(2001)\end{array}$ & Witness & \multirow{4}{*}{$\begin{array}{c}\text { Sistemas de } \\
\text { Transporte } \\
\text { Y } \\
\text { Distribució } \\
\mathrm{n}\end{array}$} \\
\hline $\begin{array}{c}\text { Reyes, E. et al. } \\
\text { (2007). }\end{array}$ & ProModel & \\
\hline $\begin{array}{c}\text { Alfaro Pozo, R., y } \\
\text { Perpiñán Pérez, L. } \\
\text { (2010). }\end{array}$ & Arena & \\
\hline $\begin{array}{c}\text { Montoya, R. et al. } \\
\text { (2011). }\end{array}$ & Arena & \\
\hline $\begin{array}{c}\text { Hernández, A. y } \\
\text { Gutiérrez, I. (2012). }\end{array}$ & Witness & \\
\hline $\begin{array}{c}\text { López, E. et al. } \\
\text { (2013). }\end{array}$ & Arena & \\
\hline $\begin{array}{c}\text { Torres, D. et al. } \\
\text { (2013). }\end{array}$ & ProModel & \\
\hline $\begin{array}{c}\text { Riascos, A. et al. } \\
\text { (2013). }\end{array}$ & ProModel & \\
\hline $\begin{array}{c}\text { Muñoz F. y Aldana, } \\
\text { E. (2005). }\end{array}$ & Ithink & \\
\hline $\begin{array}{c}\text { Caro, E. et al } \\
(2008) .\end{array}$ & Siminv & $\begin{array}{l}\text { Proyectos } \\
\text { de }\end{array}$ \\
\hline Gómez, A. (2010). & Arena & \\
\hline $\begin{array}{c}\text { Correa, L. y } \\
\text { Dueñas, D. (2011). }\end{array}$ & ProModel & \\
\hline
\end{tabular}

\section{METODOLOGÍAS}

La realización de un estudio de simulación requiere de una serie de pasos básicos, aunque en diferentes ocasiones, dependiendo de la problemática, es posible suprimir algunos o no utilizar todos. Según Guash, A. et al. (2005), la metodología para un estudio de simulación se basa en: 
(A) Definición del problema y planificación del estudio. En esta etapa, se definen los objetivos perseguidos por el planteamiento del problema. Si estos objetivos no se definen con claridad, existirá el peligro de no abordar correctamente el problema, para el cual se ha solicitado la técnica de simulación, como vía para su posible solución.

(B) Recogida de datos. La recopilación de datos e información deben ser de fuentes creíbles, y a su vez, cuestionables. Como en el campo de trabajo esto sucede muy poco, se debe responder a las preguntas planteadas del problema, realizando hipótesis razonables en colaboración con el usuario final.

(C) Formulación del modelo conceptual. Conocidos los objetivos del problema, existirá la posibilidad (tentación) de comenzar la construcción del modelo de simulación inmediatamente en el computador. Ello conduce a obtener modelos de difícil mantenimiento. Es por esto que se recomienda plantear un modelo conceptual con un nivel de abstracción superior al planteado en el código, este contemplará las relaciones estructurales más importantes del sistema y la coordinación de la sucesión de actividades que ocurrirán.

(D) Construcción del modelo. Para esto se plantearán varios modelos simplificados que caractericen las partes más esenciales del sistema, para así avanzar rápidamente a la consecución de los objetivos planteados y de esta manera, obtener un posible modelo general (Se dice "posible", ya que un modelo tiene que estar en constante mantenimiento $y$ verificación).

(F) Verificación, validación y pruebas. Esta es una de las etapas más importantes en la técnica de la simulación, ya que con una buena cantidad de pruebas, se verificará que el modelo se ejecuta correctamente y según las especificaciones (modelo conceptual), se validará que las hipótesis planteadas, la teoría empleada y otras suposiciones son correctas.
Esto favorece que nuestro modelo tenga un comportamiento lo más parecido posible a la realidad.

(G) Diseño de los experimentos de simulación. Se decidirá qué características del sistema se mostrarán a través de las estadísticas, la duración de cada simulación, las condiciones iniciales y el número de ejecuciones (réplicas); para así, inferir y tomar decisiones con mayor seguridad.

(H) Análisis de los resultados. Se estudiarán los resultados obtenidos, observando carencias y mejoras, realizando un estudio de sensibilidad de las variables de estado.

(I) Documentación e implementación. En las etapas precedentes, es muy importante mantener un documento que muestre el estado del proyecto de simulación, su evolución en paralelo de cada etapa. La implementación consistirá en tomar decisiones como fruto del estudio de simulación realizado (Ver Fig. 1).

\section{CASO DE ESTUDIO}

En el presente caso de estudio, se tomó una empresa de servicio de inspección y de laboratorio de productos petroquímicos y petróleos, cuya misión es la de suministrar la más alta calidad de servicios en los campos de inspección de transferencias en custodia, calibración de medidores y probadores, aforo de todo tipo de tanques de almacenamiento y análisis de calidad en crudo e hidrocarburos.

El objetivo principal es diseñar un modelo de simulación en ProModel que represente el comportamiento de las etapas del proceso de pruebas de metales "sílice y aluminio" en hidrocarburos en la empresa, con el fin de generar una propuestas de mejora a futuro. 


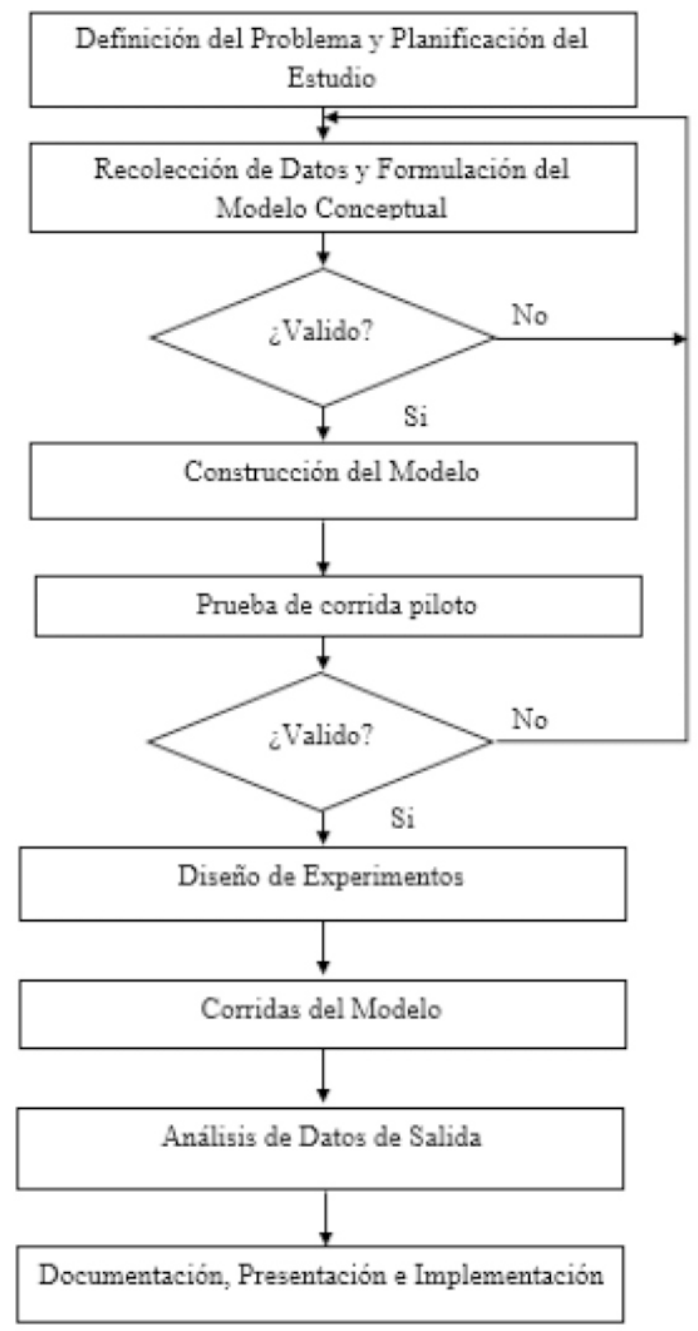

Figura 1. Metodología para el estudio de la simulación. Fuente: Guash et al. (2005).

El modelo de simulación tiene como alcance las etapas del proceso de pruebas en hidrocarburos, los cuales se identifican como análisis de control de calidad en los ensayos o pruebas de metales realizadas. El proceso escogido para esta investigación es el de metales en sílice y aluminio, puesto que en los últimos meses, la demanda de este servicio ha aumentado y debido a esto, se han presentado demoras en las entregas de los resultados

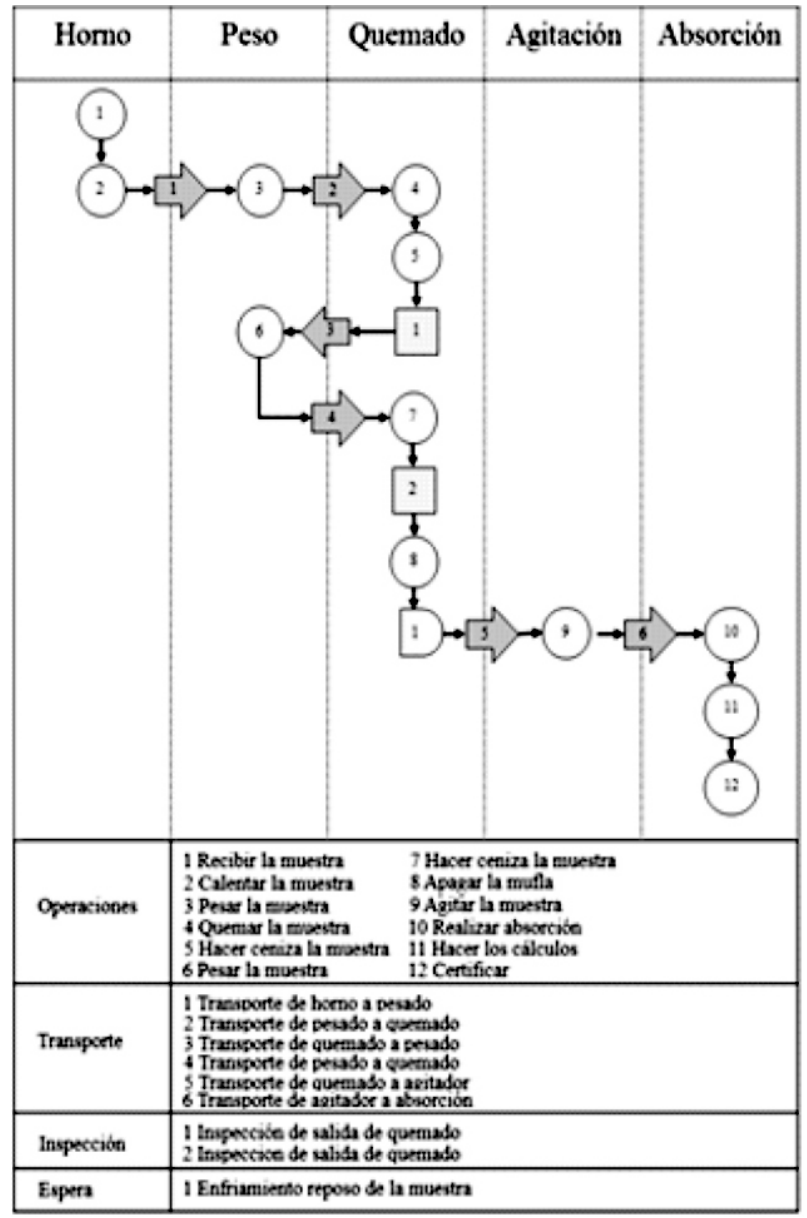

Figura 2. Diagrama de flujo del proceso..

\subsection{Descripción del proceso}

Una vez llegadas las muestras al laboratorio por parte de los clientes, se procede a realizar las siguientes etapas del proceso:

Se recibe la muestra, se calienta la muestra en un horno a temperatura de $60^{\circ} \mathrm{C}$, se pesa la muestra, se transporta al área de quemado, se hace ceniza la muestra en el equipo de mufla a una temperatura de $550^{\circ} \mathrm{C}$, se inspecciona la muestra, se pesa nuevamente en la balanza, se envían las muestras a la mufla nuevamente a una temperatura de $950^{\circ} \mathrm{C}$, se inspecciona la muestra, se apaga la mufla para dejar enfriar por un tiempo de 20 minutos, se envían las 
muestras al plato agitador, se pasa al equipo de absorción atómica para la lectura, se hacen los cálculos, certificación y envío de resultados (Ver Fig. 2).

\subsection{Modelo Conceptual}

En esta etapa, se definieron las particularidades y las medidas de desempeño a evaluar del modelo. Las características particulares para el modelo que se desarrolló se describen a continuación:

El sistema está conformado por nueve (9) locaciones: una (1) de recepción, una (2) de balanza, una (1) de quemado, una (2) de mufla, una (1) de plato agitadory calentador, una (1) de equipo de absorción atómica y una (1) de cálculo de resultados y envío.

Otro de los elementos son las entidades, que se encuentran de tres tipos: (1) Muestra, que es la cantidad representativa de hidrocarburos y derivados de un tanque o pozo que llega al laboratorio en recipientes de plástico, metal o vidrio para sus respectivos análisis; (2) producto en proceso, que es una cantidad representativa de la muestra que se toma para realizar el respectivo análisis, al quemarse se convierte en cenizas y después se funde a $950{ }^{\circ} \mathrm{C}$; producto terminado, que son los resultados de las lecturas obtenidas en el equipo de absorción atómica, los cuales son enviados vía correo electrónico a los clientes en un certificado de calidad.

Los tiempos de transporte desde la primera locación y entre las locaciones fueron despreciables.

Al sistema arriban las muestras a la primera locación denominada "Recepción".

Las medidas de desempeño que se evaluaron se indican a continuación:

Productos terminados (PT). Por medio de este indicador, se obtiene el porcentaje de muestras terminadas en el laboratorio de la empresa; esta medirá la capacidad de producción.

Tiempo de ciclo (TC). Se obtiene la cantidad de tiempo en la que permanecen las muestras durante el proceso.

Throughput (TH). Deja ver por medio del modelo, la cantidad de muestras producidas durante un tiempo determinado.

Productos en procesos (WIP). Indica la cantidad de muestras que quedan en el sistema.

\subsection{Recolección de Datos}

Se elaboró un estudio de premuestra para cada locación del modelo, teniendo en cuenta la variable correspondiente a los tiempos de operación, en unidades de minutos; para ello, se tomaron 32 datos de cada uno. A partir de estas premuestras, se calcularon las medidas de tendencia central y de dispersión. Con un nivel de confianza de $90 \%$ y un error típico de $10 \%$, se obtuvieron los respectivos tamaños de muestra para cada una de las locaciones aplicando la siguiente fórmula:

$$
\mathrm{n}=\frac{\sigma^{2} \mathrm{Z}_{\alpha / 2}^{2}}{\mathrm{E}^{2}}
$$

$\sigma^{2}$ es la varianza encontrada en los 32 datos tomados en la premuestra, $Z^{2} \alpha / 2$ corresponde al valor normalizado del nivel de confianza del estudio al cuadrado y $E^{2}$ es el error máximo esperado en el cálculo. En la Tabla 3, se detallan los resultados calculados para el tamaño de muestra de cada una de las locaciones. Se puede observar que del tamaño de la muestra depende en gran medida de la varianza de los datos, por tanto, para variabilidades altas, se encontrarán tamaños de muestra grande. 
Tabla 3. Tamaños de muestra por locaciones.

\begin{tabular}{|c|c|c|}
\hline Locación & Varianza & $\begin{array}{c}\text { Tamaño } \\
\text { Muestra }\end{array}$ \\
\hline Recepción & 0,0379 & 10 \\
\hline Balanza & 0,0444 & 12 \\
\hline Quemado & 0,1745 & 47 \\
\hline Mufla & 0,2660 & 72 \\
\hline $\begin{array}{c}\text { Plato agitador y } \\
\text { calentador }\end{array}$ & 0,2073 & 56 \\
\hline Absorción atómica & 0,3137 & 85 \\
\hline $\begin{array}{c}\text { Cálculo de registro y } \\
\text { envío de resultados }\end{array}$ & 0,3450 & 93 \\
\hline
\end{tabular}

El procedimiento de validación consta de dos fases: la primera, aplicando las pruebas de independencia y uniformidad para otorgarles un buen grado de validez a los datos. En esta fase, se encontró que todos los datos tomados satisfacen las pruebas de uniformidad e independencia. La segunda parte consiste en determinar la distribución que mejor se asemeja a los datos. Ambas fases fueron apoyadas por la herramienta Stat:Fit, perteneciente al paquete de ProModel (Ver Tabla 4).

Tabla 4. Distribución sugerida por Stat:Fit.

\begin{tabular}{|c|c|}
\hline $\begin{array}{c}\text { Variable Tiempo } \\
\text { Operación }\end{array}$ & $\begin{array}{c}\text { Distribución de la } \\
\text { probabilidad }\end{array}$ \\
\hline Recepción & $\mathrm{N}(1.45,83 \mathrm{e}-002)$ \\
\hline Balanza & Loglogistic(22.4,3.4,085) 0814$)$ \\
\hline Quemado & Weibull (261,1.44,0.811) \\
\hline Mufla & $\begin{array}{c}\text { InverseWeibull } \\
(13.2,0.955,12.1)\end{array}$ \\
\hline $\begin{array}{c}\text { Plato agitador y } \\
\text { calentador }\end{array}$ & $\begin{array}{c}\text { Logistic }(23.4,0.307) \\
\text { Equipo de absorción } \\
\text { atómica }\end{array}$ \\
\hline $\begin{array}{c}\text { Cálculo de resultados y } \\
\text { envío }\end{array}$ & $\begin{array}{c}\text { Powerfuntion } \\
(12.3,14.1,0.894)\end{array}$ \\
\hline
\end{tabular}

\subsection{Construcción del Modelo}

Para la creación del modelo, se empleó el software de simulación profesional ProModel.
El primer paso en la construcción del modelo fue ingresar la información requerida en los módulos empleados: Locations, Entities, Processing, Arrivals, Attributes, Variables, General Information y Background Graphics. (ProModel Corporation, 1999). Para plasmar la lógica del proceso de los programas, se emplearon comando de programación y palabras reservadas del software, que permitieron representar las características deseadas en cada modelo (Ver Fig. 3).

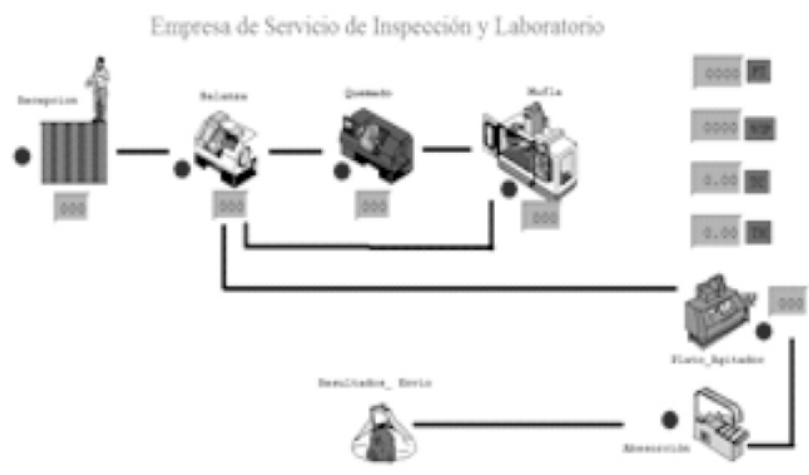

Figura 3. Modelo de simulación del proceso.

\subsection{Análisis de resultados de salida}

Realizada la simulación, la locación que tiene mayor porcentaje de ocupación es la Mufla en un 87,10\%, que equivale a 6,96 horas; seguido del Plato Agitador, en un $18,12 \%$, lo cual equivale a 1,44 horas. Las demás locaciones se encuentran por debajo de este porcentaje, lo que evidencia la mayor parte de su tiempo desocupado, por tanto, no se encuentran operando en su máxima capacidad (Ver Tabla 5).

Tabla 5. Utilización de las máquinas (\%).

\begin{tabular}{|c|c|}
\hline Locación & Porcentaje \\
\hline Recepción & $1,54 \%$ \\
\hline Balanza & $4,69 \%$ \\
\hline Quemado & $12,97 \%$ \\
\hline Mufla & $87,10 \%$ \\
\hline $\begin{array}{c}\text { Plato agitador y } \\
\text { calentador }\end{array}$ & $18,12 \%$ \\
\hline $\begin{array}{c}\text { Equipo de absorción } \\
\text { atómica }\end{array}$ & $17,45 \%$ \\
\hline $\begin{array}{c}\text { Cálculo de resultados y } \\
\text { envío }\end{array}$ & $8,26 \%$ \\
\hline
\end{tabular}


De acuerdo al análisis de las variables o indicadores de salida:

La variable PT tiene una proporción de tres (3) unidades (muestras) de producto terminado en ocho horas.

La variable WIP muestra en promedio siete (7) unidades (muestras) productos en procesos en ocho horas.

La variable TC indica la cantidad de tiempo de permanencia de las muestras durante el proceso, equivalente a 466,11 minutos durante un tiempo total de ocho horas.

La variable $\mathrm{TH}$ : esta variable presenta que las muestras producidas son de $0,01 \%$, las cuales son generadas por el sistema en una velocidad de ocho horas.

Dado lo anterior, se evidencia que la locación Mufla es el cuello de botella del proceso y sobresalta la mayor proporción de productos en proceso en comparación con los productos terminados. Así, se presentan indicios para proponer un escenario de mejora, donde exista una locación que pueda agilizar el proceso y la entrega de los resultados a los clientes.

\subsection{Modelo propuesto}

Teniendo en cuenta el análisis de los resultados, la propuesta de mejora va encaminada a cumplir con la demanda de este servicio y a reducir las demoras que se están presentado en las entregas de los resultados (Ver Fig. 4).

Por medio del modelo de simulación anterior, se logró demostrar la necesidad de mejorar el proceso donde se hace ceniza la muestra llamado Mufla y aparte de esto, mejorar la capacidad de la operación de pesado. De esta forma, se incrementará las unidades producidas y se reducirán las muestras de productos de proceso.

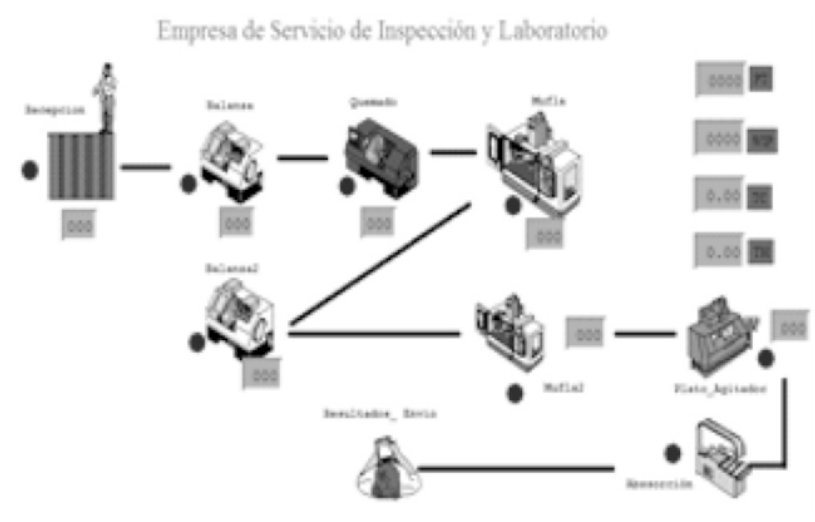

Figura 4. Modelo de simulación del propuesto.

Por medio de los indicadores, se analiza que al modelo actual, al realizarle un plan de mejora, aumenta en la variable PT de tres muestras a ocho en el modelo de dos muflas. De esta manera, también cabe resaltar que la variable WIP disminuye de siete muestras en proceso a cero muestras en proceso en el modelo de simulación de mejora (Ver Tabla 6).

Tabla 6. Comparación de los indicadores de salidas.

\begin{tabular}{|c|c|c|}
\hline Indicadores & $\begin{array}{c}\text { Modelo } \\
\text { Actual }\end{array}$ & $\begin{array}{c}\text { Modelo } \\
\text { Mejorado }\end{array}$ \\
\hline $\begin{array}{c}\text { Productos terminados } \\
\text { (PT) }\end{array}$ & 3 & 8 \\
\hline $\begin{array}{c}\text { Productos en procesos } \\
\text { (WIP) }\end{array}$ & 7 & 0 \\
\hline Tiempo de ciclo (TC) & 466,11 & 505,06 \\
\hline Throughput (TH) & 0.01 & 0,01 \\
\hline
\end{tabular}

\section{CONCLUSIONES}

Una vez realizado este trabajo investigativo, se concluye que la herramienta de simulación con ProModel no da soluciones a los problemas encontrados en el sistema, ya que esta únicamente permite conocer más el proceso, ver de manera visual y estadística el comportamiento de un sistema.

Mediante la simulación, se detectó que donde se genera el cuello de botella es en el subproceso de Mufla, en el cual se busca que las pruebas alcancen 
temperaturas altas para cumplir con los diferentes procesos que requieren este tipo de característica dentro de los laboratorios.

Se evidencia, mediante el software ProModel, que el comportamiento actual del proceso genera demoras en los tiempos de entrega de pruebas de metales de sílice y aluminio dentro de la empresa; esto se ve reflejado en los atrasos en las entregas de los pedidos solicitados por el cliente y los bajos niveles del servicio.

Una vez analizado ambos modelos, tanto el actual como el mejorado, teniendo en cuenta los resultados arrojados y los indicadores asociados a Productos Terminados, Productos en Procesos, Tiempo de Ciclo y el Throughput, se concluye que los productos terminados tiene una tasa de crecimiento correspondiente a un 166,66\%; los productos en procesos decrecen en su totalidad y el tiempo de ciclo aumenta en un $8,35 \%$.

Dado lo anterior, se le recomienda a la empresa de servicio de inspección y de laboratorio de productos petroquímicos y petróleos, la adquisición de un nuevo equipo de Mufla para mejorar la calidad de sus procesos y servicios.

\section{REFERENCIAS}

Acosta, E. (2012). Simulación en Tiempo Discreto de un Proceso de Abastecimiento de Combustible como una Herramienta de Toma de Decisiones: Caso estación de servicios en Barranquilla. Universidad Libre. Barranquilla.

Alfaro Pozo, R., y Perpiñán Pérez, L. (2010). Modelado y Simulación de un Almacén Distribuidor de Equipos de Medida Energética. Universidad Politécnica de Catalunya. España.

Álvarez Pomar, L., y Mejía, G. (2005). Caracterización, Modelamiento y Simulación de un Sistema Preferencial de Atención en un Ambiente Multiservicio. Universidad de los Andes. Bogotá.

Álvarez, S., García, F., Fernández, M., y González, P. (2001). "Aplicaciones de la Simulación en la Gestión de un Servicio de Urgencias Hospitalario". Emergencias 13, 90-96.

Arancibia Vallejos, C. (2012). Mejoramiento de Productividad Mediante Distribución de Instalaciones y Reasignación de Personal en un Área de la Planta en Empresa Textil. Universidad de Chile.

Aspray, W. y Neumann, J. (1990). The Origins of Modern Computin. Massachusetts: The MIT Press. p. 110-113.

Ávila, H. y Vásquez, M. (2008). Aplicación de Software de Simulación como Herramienta en el Rediseño de Plantas de Producción en Empresas del Sector de Alimentos. Revista Prospectiva. Vol. 6. No. 2.

Banks, J., Carson J. y Nelson, B. (1996). "Discrete - Event System Simulation". Pearson Education India.

Barceló, J. (1996). Simulación de Sistemas Discretos. Barcelona: Isdefe.

Borgetti, S. (2013). "Simulación De Procesos A Través Del Análisis De Líneas De Espera Para La Optimización De Tiempos". Revista Aristas: Ciencia e Ingeniería. Facultad de Ciencias Químicas e Ingeniería. UABC.

Caro, E., García, D., Collado, A. y del Sol, J. (2008). “Análisis de Riegos en Proyectos de Inversión Utilizando el Método de la Simulación". Observatorio de la Economía Latinoamericana 99. 
Correa, L. y Dueñas, D. (2011). "Hacia el uso de la Simulación como Herramienta para el Análisis de Proyectos de Inversión". Revista De Investigación, Desarrollo e Innovación, Vol. 1, No. 2. pp. 40-52

Coss, R. (2003). Simulación un Enfoque Práctico. México: Editora Limusa..

Cruz, M., Sánchez, M., Cueto, M., y Palafox, C. (2013). "Aplicación de Simulación para el Incremento de la Productividad de una Empresa Generadora de Panela en la Ciudad de Tuxtepec". Revista De La Ingeniería Industrial, 7(1), pp. 13-20.

De la Fuente García, D., Diez, R., Priore, P., Fernández, N., Gómez, A. G., García, F., y Quesada, I. (2002). “Simulación y Optimización de la Planificación de un Proceso Continuo: Aplicación a un Tren de Laminación". En II Conferencia de Ingeniería de Organizació,. pp. 987-994.

Díaz, J., Cubillos, J., y Fernández, M. (2010). “Metodología para la Simulación de Centros de Llamadas: Caso de estudio". Revista Studiositas". Vol. 5 No. 3, pp. 117-136.

Flores, F., y Bernardi, B. (2007). "Aplicación de la Metodología de Simulación para Sincronizar el Flujo del Producto en una Planta Tipo A con Componentes Compartidos por Medio de la TOC". Unidad Profesional Interdisciplinaria de Ingeniería y Ciencias Sociales y Administrativas. México.

García, E. García, H y Cárdenas, L. (2006). Simulación y Análisis de Sistemas con Promodel. Ciudad de México: Editorial Pearson.

Gigch, J. (1974). Applied General Systems Theory. New York: Harper and Row.

Gómez, A. (2010). "Simulación de Procesos Constructivos". Revista Ingeniería de construcción, 25(1), pp. 121-141.

Gordon, G. (1978). "Simulación de Sistemas". Ciudad de México: Editora Diana.

Guasch, A., Piera, M., Casanovas, J., y Figueras, J. (2002). Modelado y Simulación, Aplicación a Procesos Logísticos de Fabricación y Servicios. Barcelona. Universidad Politécnica de Catalunya.
Guash, A. Piera, M. Casanovas, J. y Figueras J. (2005). Aplicaciones a procesos logísticos de fabricación y servicios. Universidad Politécnica. de Catalunya. Barcelona

Hernández, A. y Gutiérrez, I. (2012). “Optimización de Infraestructuras de Transporte Combinado Mediante Simulación Iterativo: Estudio de uno Terminal trencarretero". Dyna, 87(4), pp. 474-480.

Hernández, D. (1977). Técnicas de Simulación en los Procesos de Planificación. Editorial. Instituto de Estudios de Planificación. Primera edición. España.

Hoeger, H. (2000). “Apuntes de Simulación”. Disponible en Guías en Línea:

http://webdelprofesor.ula.ve/ingenieria/hhoeger/

López, E., Casas, L., y Cajahuanca, C. (2013). “Optimización Basada en Simulación en un Sistema de Tránsito Público Masivo". Eleventh LACCEI Latin American and Caribbean Conference for Engineering and Technology (LACCEI'2013). Mexico.

Lugo Ruíz, A. y Pinzón Blanco, D. (2011). "Estrategias de Mejoramiento de los Procesos de Manufactura en Aceros y Aluminios CIA. SA". Universidad Libre. Bogotá.

Maisel, H. y Grugnoli, G. (1972). Simulation of Discrete Stochastic Systems. Chicago: Science research associates.

Montoya, J. (2007). Notas Técnicas Simulación Conceptos Básicos sobre Simulación de Eventos Discretos. Bogotá: Pontificia Universidad Javeriana.

Montoya, R., Alzate, J., y Muñoz, J. (2011). "Análisis de la Operación Despacho en un Centro de Distribución Basado en Gestión de Procesos y Simulación". INGE CUC, 7(1), $75-$ 86.

Moraga Rebolledo, M., y Baesler Abufarde, F. (2001). “Uso de Inteligencia Artificial para la Optimización de un Modelo de Simulación Aplicado a un Proceso de Remanufactura de Pino Radiata". Maderas. Ciencia y tecnología, 3(1-2), 52-62.

Moran Pacheco, R. y Torres Fuchslocher, C. (2008). “Diseño y Análisis del Proceso de Fabricación de Ventanas en Aluminios del Maule SA". Universidad de Talca. Chile. 
Muñoz F. y Aldana, E. (2005). "Modelamiento Dinámico del Sistema General de Pensiones Aplicado al Ahorro Individual". Universidad de los Andes. Bogotá.

Naylor, B. y Kong, Ch. (1971). Técnicas de Simulación en Computadoras. Editorial Limusa. México, DF, 34-78.

Nieto Buitrago, D. y Osorio Zambrano, K. (2012). Logística de Servicios en Bancos. Bogotá: Universidad del Rosario.

Paternina, C., Moreno Ibáñez, P., Montoya Torres, J., y Isaza Betancourt, C. (2011). "Simulación del Sistema de Recepción de Llamadas en una Empresa de Servicios". Revista Científica Ingeniería y Desarrollo, (8), 99-110.

Portilla, L. M., Montoya, L. A., y Henao, S. A. F. (2010). "Análisis de Líneas de Espera a través de Teoría de Colas y Simulación". Scientia Et Technica, 17(46), pp. 56-61.

Prawda, J. (1980). Métodos y Modelos de Investigación de Operaciones Vol. 2. Ciudad de México: Editora Limusa.

ProModel Corporation. (1999). ProModel, Software de Simulación de Manufactura. Estados Unidos: Manual del Usuario.

Racero, J. (2004). Ingeniería Industrial - Simulación de Eventos Discretos. Sevilla: Universidad de Sevilla Grupo de Investigación Tecnologías de la información e informática. http://racero.us.es/

Restrepo, E., Medellín, A., Ceballos, C., y Fernández, C. (2011). "Guía Metodológica para la Aplicación de un Modelo de Simulación Discreta en el Sector del Servicio Automotriz, Caso específico: Euroautos Ltda.-Renault Minuto". Universidad Pontificia Bolivariana (UPB). Medellín.

Reyes, E., Sánchez, M., y Lasserre, A. (2007). “Optimización de las Rutas de Reparto de Helado de la Empresa Fricongelados Citlaltépetl". Revista de la Ingeniería Industrial Revista de la Ingeniería Industrial ISSN, Vol. 1940, pp. 2163.

Riaño, G. (2007). "Análisis de Colas para el Diseño de una Cafetería Mediante Simulación de Eventos Discretos". Revista de Ingeniería. Universidad de los Andes. Bogotá.

Riascos, A., Gallego, S., y Orozco, G. (2013). "Sistema de Recolección de Residuos Agrícolas Cañeros en un Ingenio del Valle del Cauca. Modelado y Costeo". Universidad Nacional de Colombia (UNAL). Bogotá.

Rojas, L. y Herrera, L. (2008). "Análisis del Proceso de Urgencias y Hospitalización del CAMI Diana Turbay A Través de un Modelo de Simulación con Arena 10.0 para la Distribución Óptima del Recurso Humano". Ingeniería e Investigación, 28(1), pp. 146-153.

Saucedo Solorio, J. (2013). “Aplicación de Simulación para Evaluar la Planeación Estratégica de Producción en una Empresa del Sector Automotriz". Universidad Autónoma del Estado de Hidalgo (UAEH). España.

Shannon, R. (1988). Simulación de Sistemas. Diseño, desarrollo e implementación. Ciudad de México: Editora Trillas.

Shannon, R. (1975). "System Simulation: The Art and Science". Englewood Cliffs. Editorial Prentice-Hall.

Soto, L. (2011). "Simulación de un Sistema de Manufactura Flexible con Redes de Petri Coloreadas". Tecnología en Marcha, 23(1), p. 47.

Taddei Bringas, J., Rodríguez Carvajal, R., y Ruiz Duarte, J. (2013). "Mejora del Proceso de Inscripciones en una Institución de Educación Superior mediante Simulación". Ingeniería Industrial, 34(1), pp. 12-25.

Thierauf, R. y Grosse, R. (1976). Toma de Decisiones por Medio de Investigación de Operaciones. Ciudad de México: Editorial Limusa.

Torres, D., Hoyos, J., Villegas, M., y Fernández Ledesma, J. (2013). "Modelo de Simulación y Optimización Logística". Revista Ingeniería Industrial,1(1), 9-26.

Wiston, W. (2008). "Investigación de Operaciones Aplicaciones y Algoritmos". Grupo Editorial Iberoamericana. Cuarta edición. México. 